OPEN ACCESS

Edited by:

Izabel Galhardo Demarchi,

Federal University of

Santa Catarina, Brazil

Reviewed by:

Diego Luis Costa,

University of São Paulo, Brazil

Paulo Marcos Matta Guedes,

Federal University of Rio Grande do

Norte, Brazil

*Correspondence:

Lucas P. Carvalho

carvalholp76@gmail.com

Specialty section: This article was submitted to

Parasite and Host,

a section of the journal

Frontiers in Cellular and

Infection Microbiology

Received: 13 January 2021

Accepted: 16 March 2021

Published: 09 April 2021

Citation:

de Lima CMF, Magalhães AS,

Costa R, Barreto CC, Machado PRL,

Carvalho EM, Lessa MM and

Carvalho LP (2021) High Anti-

Leishmania IgG Antibody Levels

Are Associated With Severity

of Mucosal Leishmaniasis.

Front. Cell. Infect. Microbiol. 11:652956.

doi: 10.3389/fcimb.2021.652956

\section{High Anti-Leishmania IgG Antibody Levels Are Associated With Severity of Mucosal Leishmaniasis}

\author{
Clara Mônica F. de Lima ${ }^{1,2}$, Andrea S. Magalhães ${ }^{1}$, Rúbia Costa ${ }^{1}$, \\ Carolina C. Barreto ${ }^{1}$, Paulo R. L. Machado ${ }^{1,2,3}$, Edgar M. Carvalho ${ }^{1,2,3,4}$, \\ Marcus M. Lessa ${ }^{1,2}$ and Lucas P. Carvalho ${ }^{1,2,3,4^{*}}$ \\ 1 Serviço de Imunologia, Hospital Universitário Prof. Edgard Santos, Universidade Federal da Bahia, Salvador, Brazil, \\ 2 School of Medicine, Programa de Pós-graduação em Ciências da Saúde - Universidade Federal da Bahia, Salvador, Brazil, \\ ${ }^{3}$ Ministry of Sciences and Technology, Instituto Nacional de Ciência e Tecnologia em Doenças Tropicais, INCT-DT, \\ Salvador, Brazil, ${ }^{4}$ Laboratório de Pesquisas Clínicas (LAPEC), Instituto Gonçalo Moniz (IGM), Fiocruz, Salvador, Brazil
}

Background: Mucosal leishmaniasis (ML), the most inflammatory form of tegumentary leishmaniasis, is predominantly caused by Leishmania braziliensis. The disease is characterized by the development of lesions, mainly in the nasal mucosa. An exacerbated inflammatory response has been associated with the presence of destructive and disfiguring lesions, with stages of severity ranging from small nodulations to the complete destruction of the nasal pyramid architecture. As Leishmania is an intracellular parasite, most immunological studies have emphasized the cell-mediated immune response, while relatively few studies aimed to investigate the role antibodies in protection against, or the pathology of $\mathrm{ML}$.

Methods: Patients with a confirmed diagnosis of ML were classified according to clinical staging criteria. Serum levels of Leishmania-specific lgG, IgG1 and IgG2 antibodies were determined by ELISA before and after treatment with antimony or antimony plus pentoxifylline.

Results: Patients in stages IV and V produced higher concentrations of IgG and IgG1 antibodies when compared to those in stage I and II. Significant reductions were seen in the concentrations of $\lg G$ and $\lg G 2$ antibodies in most patients who responded well to treatment.

Conclusions: Our data demonstrate an association between $\lg G$ antibody titers and the severity of mucosal disease. The observed reduction in antibody production after successful treatment in most patients preliminarily indicates that these tests can be used to aid in the assessment of therapeutic response. 


\section{INTRODUCTION}

American tegumentary leishmaniasis (ATL) is widely distributed around the world, and often presents high morbidity due to the possibility of developing destructive lesions that can disfigure and disable individuals, significantly impacting their quality of life (World Health Organization - Control of Leishmaniasis, 2010). In endemic areas of Leishmania braziliensis transmission, mucosal leishmaniasis (ML), a disease also known as mucocutaneous leishmaniasis, occurs in 3\% of patients concomitantly or following the cure of cutaneous leishmaniasis (CL). ML can also be caused by other Leishmania species, such as Leishmania amazonensis and Leishmania guaianensis (Grimaldi et al., 1987). In about $15 \%$ of cases, no previous history or scarring due to CL is documented (Boaventura et al., 2006; Miranda Lessa et al., 2007). Moreover, up to $40 \%$ of patients with an emergent disease termed disseminated cutaneous leishmaniasis (DL) have mucosal involvement (Carvalho et al., 1994). While ML primarily affects the nasal mucosa (90\% of cases), the second most affected site is the pharyngeal mucosa, followed by the laryngeal mucosal and oral cavity (Marsden, 1994; Miranda Lessa et al., 2007). Importantly, the involvement of these latter areas is an indicator of disease severity (Marsden, 1994; Miranda Lessa et al., 2007). Our group proposed staging criteria (I to V) for patients who only have nasal mucosal involvement. Stage I is characterized by nodulation in the absence of ulceration in the mucosa of the nasal septum; stage II: superficial ulceration; stage III: deep ulceration; stage IV: nasal septum perforation; stage V: destruction of the nasal pyramid architecture (Lessa et al., 2012).

As Leishmania is an intracellular parasite, the role of the innate and $\mathrm{T}$ cell responses in the pathogenesis of disease has been widely studied. Lymphocytes from ML patients were found to be more proliferative than those from CL patients when stimulated with Leishmania antigens (Carvalho et al., 2007), and the production of cytokines/chemokines by macrophages and T cells, such as TNF, IFN- $\gamma$ and CXCL9, was observed to be higher in ML compared to CL (Bacellar et al., 2002; Junqueira Pedras et al., 2003). The impairment in regulatory mechanisms that may attenuate the immune response also play an important role in the exaggerated inflammatory response observed in ML, as Cells from ML patients produce less IL-10 and its receptor than those from CL. The role of antibodies in Leishmania has not been completely elucidated. High levels of antibodies are produced in all clinical forms of leishmaniasis, and high levels of IgG and IgE antibodies are found in ML (Junqueira Pedras et al., 2003).

In the present study, we observed that antibodies against Leishmania antigens were associated with ML severity, and the data suggest that therapeutic success may be associated with decreases in antibody levels.

\section{MATERIALS AND METHODS}

\section{Study Design}

A longitudinal study was performed to investigate the association between IgG titers and disease severity, as well as response to therapy in ML patients. Clinical stages were determined upon
ENT examination and classified from stages I to $\mathrm{V}$ according to the criteria established by Lessa et al. (2012). The production of anti-Leishmania antigens antibodies was determined before and after therapy. Treatment was considered successful upon the complete re-epithelization of nasal cavity lesions (clinical cure).

\section{Study Area and Patients}

This study received approval (CAAE: 01229212.0.0000.0049) from the Institutional Review Board of the Professor Edgard Santos University Hospital (HUPES-UFBA), Salvador, BahiaBrazil. This study was carried in an area endemic for CL, Corte de Pedra, located in southeastern Bahia, Brazil. This region is home to a municipal clinic that houses a reference center for tegumentary leishmaniasis diagnosis and treatment. Diagnosis was based on the presence of nodules and ulcers in the nasal mucosa, and by the presence of Leishmania DNA as confirmed by PCR (Weirather et al., 2011). Twenty-seven ML patients were enrolled in this study, six of whom received pentavalent antimony alone $(20 \mathrm{mg} / \mathrm{Kg} /$ day for 30 days), while 21 patients were treated with a combined regimen consisting of pentavalent antimony with pentoxifylline (1200 mg/day orally for 30 days). To evaluate serological test specificity using a soluble antigen of Leishmania brazilliensis (SLA) in patients with ML, serum from 25 patients with cutaneous leishmaniasis (CL) were used as controls. Clinical and serological evaluations were performed in ML patients prior to and 90 days following the onset of therapy.

\section{Soluble Leishmania Antigen}

A L. braziliensis strain (MHOM/BR/2001), isolated from a patient with ML, was used to prepare soluble Leishmania antigen by sonication and centrifugation, as previously described (Reed et al., 1986). SLA was used at a concentration of $5 \mu \mathrm{g} / \mathrm{mL}$, after testing negative for endotoxins using the Limulus amebocyte lysate test.

\section{Detection of Anti-Leishmanial IgG Antibodies by ELISA}

Peripheral blood from ML patients was added to a dry tube for serum separation and stored at $-20^{\circ} \mathrm{C}$. Polystyrene plates were sensitized with $5 \mu \mathrm{g} /$ well of soluble Leishmania antigen in carbonate/bicarbonate buffer and incubated overnight at $4^{\circ} \mathrm{C}$. To block unspecific antibody binding, $1 \%$ bovine albumin (BSA) in PBS was added for 1 hour at $37^{\circ} \mathrm{C}$. Sera (1:50 dilution) from patients and healthy controls were added and incubated for 1 hour at $37^{\circ} \mathrm{C}$. Peroxidase-conjugated monoclonal anti-human IgG (Sigma-Aldrich, St. Louis, MO, USA), or monoclonal antihuman IgG1 and IgG2 (Sigma-Aldrich, St. Louis, MO, USA) were then added. After each step described above, 4-7 washes were performed with phosphate buffered saline containing $0.05 \%$ Tween 20. Finally, the enzyme substrate Tetramethylbenzidine (TMB) was added, and after 15 minutes the reaction was quenched with $2 \mathrm{~N} \mathrm{H}_{2} \mathrm{SO}_{4}$. Readings were performed on a microplate reader at a wavelength of $450 \mathrm{~nm}$, and results were expressed as optical density (OD). The cut off values (total IgG is 
0.395; IgG1 0.042; IgG2 0.159) were established by taking into account the mean +3 standard deviations of absorbance readings from healthy subjects.

\section{Detection of Cytokines in Serum}

Serum was obtained from peripheral blood from $15 \mathrm{ML}$ patients. Determination of IFN- $\gamma$ and TNF concentrations were performed by ELISA (BD biosciences) according to the manufactures instructions. The results are expressed in $\mathrm{pg} / \mathrm{ml}$.

\section{Statistical Analysis}

Non-parametric testing involved the Kruskal-Wallis and Wilcoxon signed-rank tests, for comparisons of continuous variables using GraphPad Prism version 5.0 (GraphPad Software, San Diego, CA, USA). Statistical differences were considered when $\mathrm{p}$ values were less than 0.05 .

\section{RESULTS}

Twenty-seven patients with ML and 25 with CL were enrolled in this study. Due to the small number of patients classified as stage I or $\mathrm{V}$, patients were divided into three groups: Stages I and II (40.7\%), stage III (25.9\%) and stages IV and V (33.4\%). Regarding sex, males were predominant in most groups, although no statistical significant difference was found between groups. We also found no difference in the frequency of individuals that reached cure within 90 days after therapy initiation, between groups. Ninety-six percent of patients with ML had previous CL lesion whereas no CL patients had history of leishmaniasis.

Titers of IgG antibodies are known to increase in active CL and visceral leishmaniasis, and decrease after cure (FagundesSilva et al., 2012). Since ML is the most inflammatory form of tegumentary leishmaniasis, we first wanted to investigate whether there were differences among IgG titers between ML and CL patients and found no differences in total IgG, IgG1 and IgG2 titers between these groups (Figure 1). We also examined antibodies levels among ML patients in different stages of disease. Interestingly, patients on stages IV and V presented high titers of total IgG and IgG2 when compared to those in stages I and II (Figure 2).

To determine whether reduced antibody production was associated with a successful therapeutic response, the titers of Leishmania-specific IgG, IgG1 and IgG2 were determined before and 90 days following the onset of therapy in $18 \mathrm{ML}$ patients. Reductions were observed in the absorbance of total IgG, in $73 \%$ of patients, and IgG2, in $89 \%$ of patients who successfully responded to antimonial treatment (Figure 3). Interestingly, increase in the levels of IgG, IgG1 and IgG2 was observed in the three individuals that failed therapy.

We have previously documented that mononuclear cells from ML patients produce high levels of IFN- $\gamma$ and inflammatory cytokines in response to Leishmania antigens (Bacellar et al., 2002). It is known that IFN- $\gamma$ induces IgG2 production and IgG induces TNF in monocytes through Fc $\gamma$ III (Kawano and Noma, 1996; Chouchakova et al., 2001; Chakraborty et al., 2021). Here we found that serum levels of IFN- $\gamma$ and TNF in ML patients are increased during disease and decreases after treatment, and a positive correlation between pre-treatment TNF and pretreatment IgG1 serum levels was observed (Figure 4).

\section{DISCUSSION}

Although ML, which predominantly affects the nasal mucosa, only occurs in less than 5\% of individuals with American tegumentary leishmaniasis, its importance is recognized by the potential to develop destructive nasal lesions, which could then spread to the face. Also, high rates of treatment failure have been documented, not only using pentavalent antimony, but also in response to other leishmanicidal drugs, such as amphotericin $\mathrm{B}$ and miltefosine (Soto et al., 2005; Machado et al., 2010). We previously showed that the combination of pentoxifylline with
A

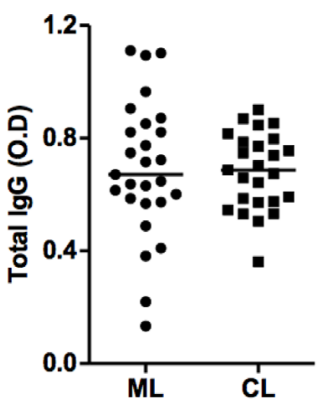

B

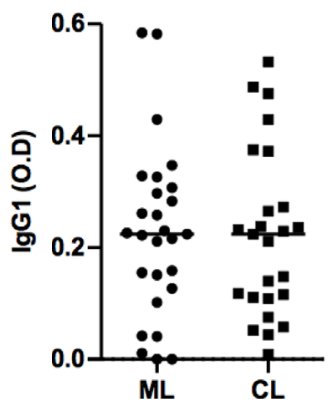

C

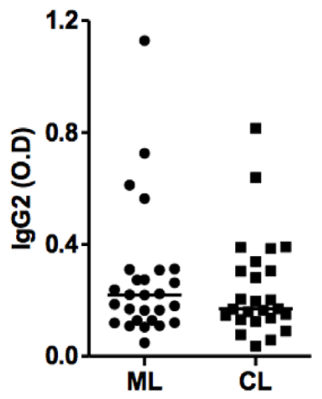

FIGURE 1 | ML and CL patients produce lgG antibodies against Leishmania braziliensis antigens. Anti-SLA total IgG (A), IgG1 (B) and IgG2 (C) titers from ML $(n=27)$ and $C L(n=25)$ patients, performed by ELISA technique. Statistical analysis was performed by the Mann-Whitney $U$ test. Results are expressed in optical density (OD). 
A

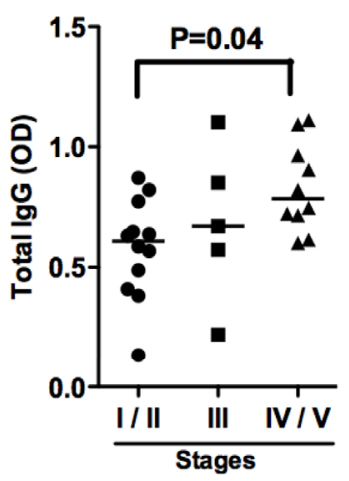

B

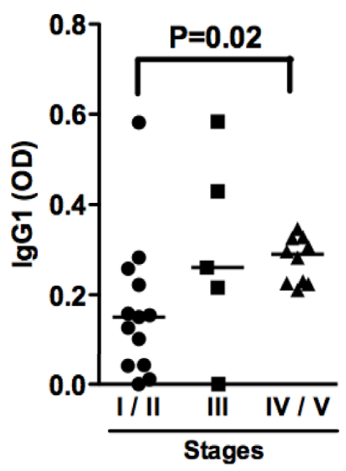

C

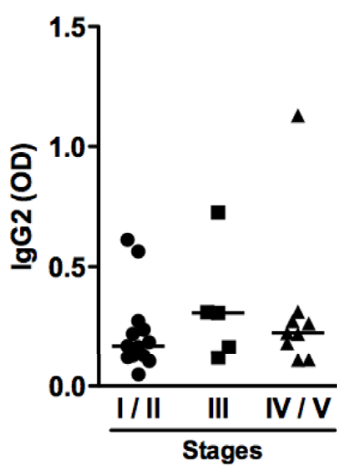

FIGURE 2 | ML patients with severe disease present increased Total lgG and lgG1 antibodies titers. Anti-SLA total lgG (A), lgG1 (B) and lgG2 (C) titers from ML patients in different stage of the disease, performed by ELISA technique. Statistical analysis was performed by the Kruskal-Wallis test. Results are expressed in optical density (OD). ML stage I/II ( $n=12) ; M L$ stage III ( $n=5) ;$ ML stage IVN ( $n=10)$.

A

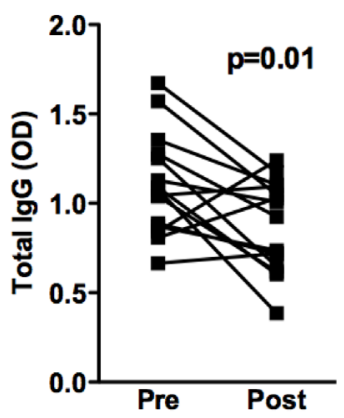

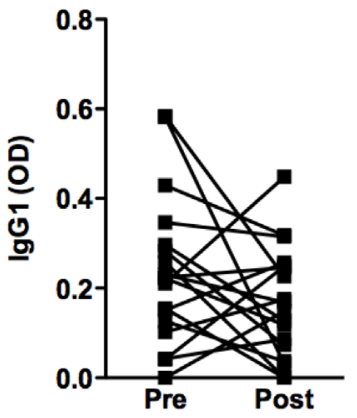

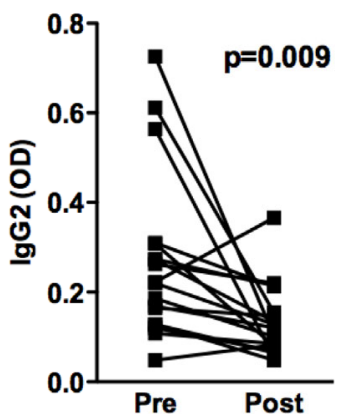

FIGURE 3 | IgG and IgG subclasses titers before and after treatment of ML patients. Anti-SLA total $\lg G(n=15)$ (A), $\lg G 1$ ( $n=18)$ (B) and lgG2 ( $n=18)$ (C) titers from ML patients, performed by ELISA technique. Statistical analysis was performed by the Wilcoxon signed-rank test. Results are expressed in optical density (OD).

A

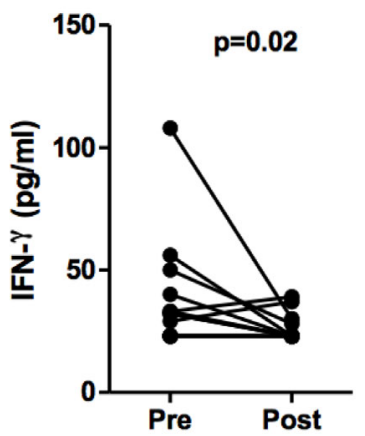

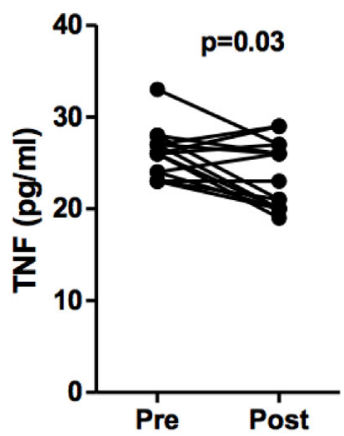

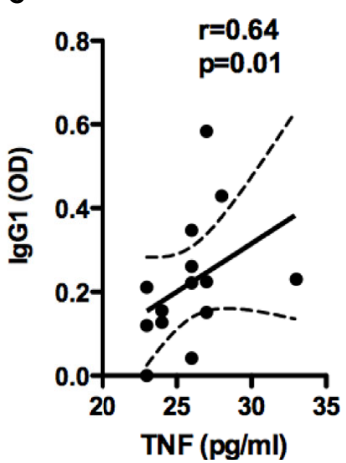

FIGURE 4 | Serum levels of IFN- $\gamma$ and TNF from ML patients. Serum concentration of IFN- $\gamma(\mathbf{A})$ and TNF (B) from ML patients $(n=15)$ were assessed before and 90 days after pentavalent antimony therapy, by ELISA technique. Statistical analysis was performed by the Wilcoxon signed-rank test. Results are expressed in pg/ml.

(C) Spearman correlation between TNF and lgG1 serum concentration, before treatment. 
antimony increases ML cure rates and cures patients refractory to antimony. The high rate of cure observed herein stands in agreement with a previous study in which pentavalent antimony in association with pentoxifylline resulted in $100 \%$ of included ML patients evolving to cure (Machado et al., 2007).

Most immunological studies in patients with tegumentary leishmaniasis place emphasis on the cell-mediated response, while few investigations have focused on the role of immunoglobulins in protection against or severity of disease. The majority of studies involving the latter area, are concerned with the use of antibodies in serological testing leishmaniasis diagnostic purposes. To investigate associations between the severity of mucosal disease and elevated levels of antibodies against soluble Leishmania braziliensis antigen, we compared titers of IgG, IgG1 and IgG2 in the sera of patients with CL and ML prior to and following the onset of treatment, but found no differences between these clinical forms.

Interestingly, we documented an association between high levels of anti-leishmania IgG and IgG1 subclass antibodies with more advanced stages of ML, which suggests either the participation of antibodies in disease severity, or that the antibody absorbance represents a marker of severity. Antibodies may participate in the pathology of leishmaniasis by way of two mechanisms: 1- Opsonization, which increases parasite uptake by macrophages and, consequently, increases parasite burden within these cells; 2- Through increased antibody-mediated cytotoxicity, i.e. higher rates of infected macrophage killing lead to the release of Leishmania antigens (Barral-Netto et al., 1995). Both mechanisms can result in increases in inflammatory response and tissue damage. IFN- $\gamma$ is known to induce IgG2 production, and IgG induces TNF through Fc $\gamma$ III (CD16). We previously documented increase in the frequency of CD16+ circulating monocytes in CL patients and found that CD16+ monocytes are the main source of TNF and IL-1 $\beta$ in CL patients (Passos et al., 2015; Santos et al., 2018). In the present work we found a positive correlation between systemic concentrations of TNF and IgG1 antibodies, suggesting a contribution of IgG1 to the deleterious inflammatory response and immunopathology observed in ML.

The lack of markers of response to treatment in cutaneous leishmaniasis continues to present challenges to successful therapeutic intervention. Here we documented a drop in the levels of IgG and IgG2 in most ML patients between 60 and 90 days after treatment initiation. Reduced IgG antibody production after cure has also been observed in patients with CL (Schubach et al., 1998; Mendonca et al., 2004). To further investigate the potential applicability of antibodies as markers of therapeutic response in ML, new studies must be conducted involving higher numbers of patients with more extensive timepoint antibody measurements.

One limitation of the present study was the small number of patients evaluated after therapy. However, we were able to clearly demonstrate higher levels of IgG and IgG1 anti-Leishmania antibodies in more advanced stages of mucosal nasal disease, in which patients have already developed deep ulcers or septal perforation. Moreover, as the levels of these immunoglobulins drop in most patients after the onset of therapy, antibody measurements may serve as a biomarker of ML prognosis, as well as be a tool capable of supporting the decision to interrupt follow-up in outpatient settings.

Although current serological diagnostic capabilities cannot differentiate between patients with $\mathrm{ML}$ and those with CL, the presently established association between antibody absorbance and clinical disease staging suggests that antibodies do indeed participate in the pathogenesis of ML. In addition, the observed reductions in antibody production after treatment and cure provides a preliminary indication that these tests may prove useful in assessing therapeutic response.

\section{DATA AVAILABILITY STATEMENT}

The datasets presented in this study can be found in online repositories. The names of the repository/repositories and accession number(s) can be found below: https://doi.org/10. 6084/m9.figshare.13415183.v1.

\section{ETHICS STATEMENT}

The studies involving human participants were reviewed and approved by Professor Edgard Santos University Hospital (HUPES-UFBA), Salvador, Bahia-Brazil. The patients/ participants provided their written informed consent to participate in this study.

\section{AUTHOR CONTRIBUTIONS}

CL, LC, ML, RC, CC, and EC conceived and designed the study. CL, LC, AM, and RC analyzed the data. CL, LC, AM, and EC interpreted the data. CL, LC, AM, RC, and CC drafted the manuscript. CL, LC, ML, AM, RC, and EC critically revised the manuscript for intellectual content. EC is guarantor of the manuscript. All authors contributed to the article and approved the submitted version.

\section{FUNDING}

This study was supported by the US National Institutes of Health grant AI-136032.

\section{ACKNOWLEDGMENTS}

We acknowledge Ednaldo Lago for providing assistance with the patient selection. The authors also thank Andris K. Walter for English language manuscript revision and copyediting services. 


\section{REFERENCES}

Bacellar, O., Lessa, H., Schriefer, A., Machado, P., Ribeiro de Jesus, A., Dutra, W. O., et al. (2002). Up-regulation of Th1-type responses in mucosal leishmaniasis patients. Infect. Immun. 70 (12), 6734-6740. doi: 10.1128/iai.70.12.6734-6740.2002

Barral-Netto, M., Barral, A., Brodskyn, C., Carvalho, E. M., and Reed, S. G. (1995). Cytotoxicity in human mucosal and cutaneous leishmaniasis. Parasite Immunol. 17 (1), 21-28. doi: 10.1111/j.1365-3024.1995.tb00962.x

Boaventura, V. S., Cafe, V., Costa, J., Oliveira, F., Bafica, A., Rosato, A., et al. (2006). Concomitant early mucosal and cutaneous leishmaniasis in Brazil. Am. J. Trop. Med. Hyg. 75 (2), 267-269. doi: 10.4269/ajtmh.2006.75.267

Carvalho, E. M., Barral, A., Costa, J. M., Bittencourt, A., and Marsden, P. (1994). Clinical and immunopathological aspects of disseminated cutaneous leishmaniasis. Acta Trop. 56 (4), 315-325. doi: 10.1016/0001-706x(94)90103-1

Carvalho, L. P., Passos, S., Bacellar, O., Lessa, M., Almeida, R. P., Magalhaes, A., et al. (2007). Differential immune regulation of activated $\mathrm{T}$ cells between cutaneous and mucosal leishmaniasis as a model for pathogenesis. Parasite Immunol. 29 (5), 251-258. doi: 10.1111/j.1365-3024.2007.00940.x

Chakraborty, S., Gonzalez, J., Edwards, K., Mallajosyula, V., Buzzanco, A. S., Sherwood, R., et al. (2021). Proinflammatory IgG Fc structures in patients with severe COVID-19. Nat. Immunol. 22 (1), 67-73. doi: 10.1038/s41590-020-00828-7

Chouchakova, N., Skokowa, J., Baumann, U., Tschernig, T., Philippens, K. M., Nieswandt, B., et al. (2001). Fc gamma RIII-mediated production of TNF-alpha induces immune complex alveolitis independently of CXC chemokine generation. J. Immunol. 166 (8), 5193-5200. doi: 10.4049/jimmunol.166.8.5193

Fagundes-Silva, G. A., Vieira-Goncalves, R., Nepomuceno, M. P., de Souza, M. A., Favoreto, S.Jr., Oliveira-Neto, M. P., et al. (2012). Decrease in anti-Leishmania IgG3 and IgG1 after cutaneous leishmaniasis lesion healing is correlated with the time of clinical cure. Parasite Immunol. 34 (10), 486-491. doi: 10.1111/ j.1365-3024.2012.01379.x

Grimaldi, G.Jr., David, J. R., and McMahon-Pratt, D. (1987). Identification and distribution of New World Leishmania species characterized by serodeme analysis using monoclonal antibodies. Am. J. Trop. Med. Hyg. 36 (2), 270-287. doi: 10.4269/ajtmh.1987.36.270

Junqueira Pedras, M., Orsini, M., Castro, M., Passos, V. M., and Rabello, A. (2003). Antibody subclass profile against Leishmania braziliensis and Leishmania amazonensis in the diagnosis and follow-up of mucosal leishmaniasis. Diagn. Microbiol. Infect. Dis. 47 (3), 477-485. doi: 10.1016/s0732-8893(03)00141-x

Kawano, Y., and Noma, T. (1996). Role of interleukin-2 and interferon-gamma in inducing production of IgG subclasses in lymphocytes of human newborns. Immunology 88 (1), 40-48. doi: 10.1046/j.1365-2567.1996.d01-634.x

Lessa, H. A., Lessa, M. M., Guimaraes, L. H., Lima, C. M., Arruda, S., Machado, P. R., et al. (2012). A proposed new clinical staging system for patients with mucosal leishmaniasis. Trans. R. Soc. Trop. Med. Hyg. 106 (6), 376-381. doi: 10.1016/j.trstmh.2012.03.007

Machado, P. R., Lessa, H., Lessa, M., Guimaraes, L. H., Bang, H., Ho, J. L., et al. (2007). Oral pentoxifylline combined with pentavalent antimony: a randomized trial for mucosal leishmaniasis. Clin. Infect. Dis. 44 (6), 788793. doi: $10.1086 / 511643$

Machado, P. R., Ampuero, J., Guimaraes, L. H., Villasboas, L., Rocha, A. T., Schriefer, A., et al. (2010). Miltefosine in the treatment of cutaneous leishmaniasis caused by Leishmania braziliensis in Brazil: a randomized and controlled trial. PloS Negl. Trop. Dis. 4 (12), e912. doi: 10.1371/ journal.pntd.0000912

Marsden, P. D. (1994). Mucosal leishmaniasis due to Leishmania (Viannia) braziliensis L(V)b in Tres Bracos, Bahia-Brazil. Rev. Soc. Bras. Med. Trop. 27 (2), 93-101. doi: 10.1590/s0037-86821994000200007

Mendonca, M. G., de Brito, M. E., Rodrigues, E. H., Bandeira, V., Jardim, M. L., and Abath, F. G. (2004). Persistence of leishmania parasites in scars after clinical cure of American cutaneous leishmaniasis: is there a sterile cure? J. Infect. Dis. 189 (6), 1018-1023. doi: 10.1086/382135

Miranda Lessa, M., Andrade Lessa, H., Castro, T. W. N., Oliveira, A., Scherifer, A., Machado, P., et al. (2007). Mucosal leishmaniasis: epidemiological and clinical aspects. Braz. J. Otorhinolaryngol. 73 (6), 843-847. doi: 10.1016/S1808-8694 (15) $31181-2$

Passos, S., Carvalho, L. P., Costa, R. S., Campos, T. M., Novais, F. O., Magalhães, A., et al. (2015). Intermediate monocytes contribute to pathologic immune response in Leishmania braziliensis infections. J. Infect. Dis. 211 (2), 274-282. doi: 10.1093/infdis/jiu439

Reed, S. G., Badaro, R., Masur, H., Carvalho, E. M., Lorenco, R., Lisboa, A., et al. (1986). Selection of a skin test antigen for American visceral leishmaniasis. Am. J. Trop. Med. Hyg. 35 (1), 79-85. doi: 10.4269/ajtmh.1986.35.79

Santos, D., Campos, T. M., Saldanha, M., Oliveira, S. C., Nascimento, M., Zamboni, D. S., et al. (2018). IL-1 $\beta$ Production by Intermediate Monocytes Is Associated with Immunopathology in Cutaneous Leishmaniasis. J. Invest. Dermatol. 138 (5), 1107-1115. doi: 10.1016/j.jid.2017.11.029

Schubach, A., Marzochi, M. C., Cuzzi-Maya, T., Oliveira, A. V., Araujo, M. L., Oliveira, A. L., et al. (1998). Cutaneous scars in American tegumentary leishmaniasis patients: a site of Leishmania (Viannia) braziliensis persistence and viability eleven years after antimonial therapy and clinical cure. Am. J. Trop. Med. Hyg. 58 (6), 824-827. doi: 10.4269/ajtmh.1998.58.824

Soto, J., Toledo, J., Vega, J., and Berman, J. (2005). Short report: efficacy of pentavalent antimony for treatment of colombian cutaneous leishmaniasis. Am. J. Trop. Med. Hyg. 72 (4), 421-422. doi: 10.4269/ajtmh.2005.72.421

Weirather, J. L., Jeronimo, S. M., Gautam, S., Sundar, S., Kang, M., Kurtz, M. A., et al. (2011). Serial quantitative PCR assay for detection, species discrimination, and quantification of Leishmania spp. in human samples. J. Clin. Microbiol. 49 (11), 3892-3904. doi: 10.1128/JCM.r00764-11

World Health Organization - Control of Leishmaniasis. (2010). WHO Technical Report Series. Iris - International repository for information sharing.

Conflict of Interest : The authors declare that the research was conducted in the absence of any commercial or financial relationships that could be construed as a potential conflict of interest.

Copyright (c) 2021 de Lima, Magalhães, Costa, Barreto, Machado, Carvalho, Lessa and Carvalho. This is an open-access article distributed under the terms of the Creative Commons Attribution License (CC BY). The use, distribution or reproduction in other forums is permitted, provided the original author(s) and the copyright owner(s) are credited and that the original publication in this journal is cited, in accordance with accepted academic practice. No use, distribution or reproduction is permitted which does not comply with these terms. 